\title{
Connected and Automated Vehicle Road Safety
}

\section{Contribution}

\author{
Philippe Chretien \\ CEESAR, European Center for Safety Analysis \& Risk Analysis, Road Safety Research Laboratory, Nanterre 92000, France
}

\begin{abstract}
Road deaths have been reduced in Europe, but progress has stopped since 2013. A shared explanation is that new technologies can increase driver mind distance to the vehicle road environment because of distraction or cockpit isolation, factors rarely declared by drivers after the accident. The connected and automated vehicle technology is under pressure as it will have to thwart this negative trend, but also to contribute to carbon dioxide emission reduction, traffic improvement, passengers comfort and pollutants reduction in towns. Our challenge is to help drivers to satisfy all these goals with the best compromise, without any distraction from close reality. The technology is capable, but safety benefits will result from positive and negative effects: recommendations to drivers or to automated vehicles are today built separately for each goal, with risk to send inconsistent recommendations through different systems: information can be a penalty for some drivers in a risky situation, especially if mental charge is too high. In a context of public mistrust on vehicle emissions, safety efficiency has to be demonstrated in risky situations before deployment, using virtual reality, based on coordinated content and validated HMI (Human Machine Interface) principles: public authorities will have to coordinate it between Europe and America.
\end{abstract}

Key words: Safety, connected, autonomous, energy, comfort, emissions, accident.

\section{Introduction}

It is now urgent to replace new mobility in its safety context, as recent technologies have not demonstrated yet their efficiency in terms of road safety.

In France [1], Fig. 1 shows that after 40 years of improvement based on public policy but also on car manufacturer contributions using CEESAR [2] support (accidentology, shock biomechanics, driver behavior), we are now facing 3 years of continuous degradation, and 2017 will be close to 2016 .

Facts are stubborn and reveal a complex reality, where human behavior has to be taken into account, more than ever.

For technical specialists, the connected mobility is a key enabler: road safety will be manageable when connected and autonomous vehicles will be generalized. This means public acceptance and limited

Corresponding author: Philippe Chretien, M.En., research fields: biomechanics, human behavior and accidentology research laboratory director. E-mail: philippe.chretien@ceesar.fr. costs, a long intermediate phase, whose road safety is worrying because of the huge diversity of vehicles situations and driver behaviors.

For connected and automated mobility, public authorities have now to anticipate all safety requirements, keeping in mind other expectations: reduce real drive emissions and fuel consumption reduction, improve traffic management, passengers comfort and worker availability for their activity.

This extended challenge leads to searching each time for each vehicle the best compromise, for example between greenhouse climate contribution and local emission in towns. The highest difficulty is probably to make connected recommendation happen in the real life, through human drivers: connected mobility will have to demonstrate road safety, and so will have to avoid driver distraction.

The connected vehicle challenge has to be analyzed globally, as it will prepare the following revolution called "autonomous vehicle". A recent automotive lesson learnt is that an implicit requirement like real 


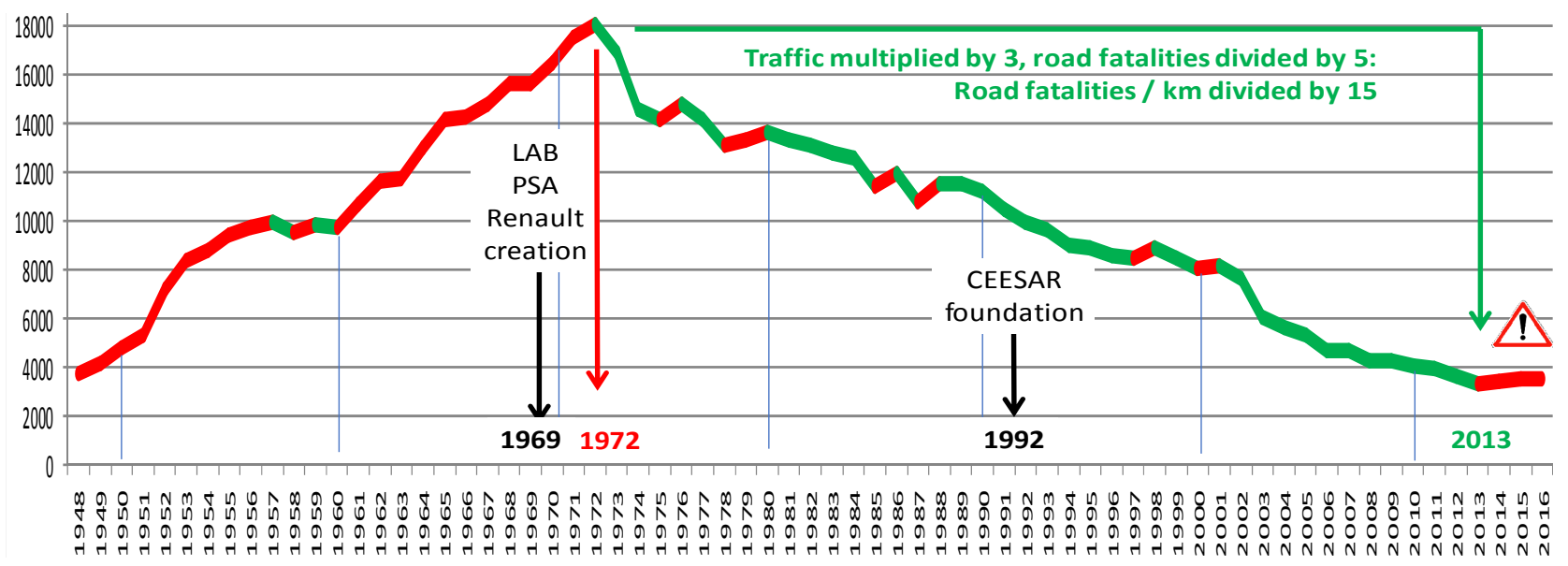

Fig. 1 Number of deaths per year on French roads.

drive emissions can become the public priority: road safety is in the same position, implicit but so important!

Road user citizens will require real life safety demonstration: that implies standards to be defined and validated in front of human behavior in risky situations, shared and applied by all vehicle and road equipment manufacturers, at worldwide level.

Under public authorities' coordination, this global work has to gather efforts in three directions: vehicles, infrastructure and road user education, with contributions from different industries and various organizations at worldwide level: a huge challenge!

\section{Regulatory Control}

Vehicle automation begins with driver delegation (called Level 3 or 4). It will be followed by full autonomous vehicle (Level 5) which is different from driver delegation, limited to private use or experimentation, under the responsibility of each country.

End of 2018, driving delegation will be regulated by the European Community, but the connected technology is only considered as an informative ADAS (advanced driver assistance system): no specific regulation is planned for connected vehicles, but a huge normative effort is required to manage such a SOS (System Of Systems), coming from separated development domains, as vehicle industry, infrastructure equipment, driving regulation and education.

The priorities today are communication and message protocols, but the system efficiency will require more: the HMI (Human Machine Interface) is crucial to get a good reaction, especially in a rental car where the driver is not familiar with vehicle specificities.

In France, the automotive network (PFA, French Automotive Patform) is looking for a common HMI policy to limit cognitive load and to help for appropriation, but the brand competition is a real difficulty to build shared normative rules: alert and display strategies are depending on brands in the French C-ITS project, named SCOOP@F [3], as dashboard design and show are important differentiation criteria between brands.

Associated with the increasing success of vehicle sharing, safety contribution of connected mobility is not guaranteed.

In a context of mistrust concerning real drive emissions, a prolongation of the road fatalities negative trend could be associated to these new technologies, with suspicion to this driver connection to distant systems: later, this suspicion to new driving technologies would be a huge penalty for automated vehicle deployment.

In such a case, citizens would be able to imagine that the complexity of real driving has been hidden 


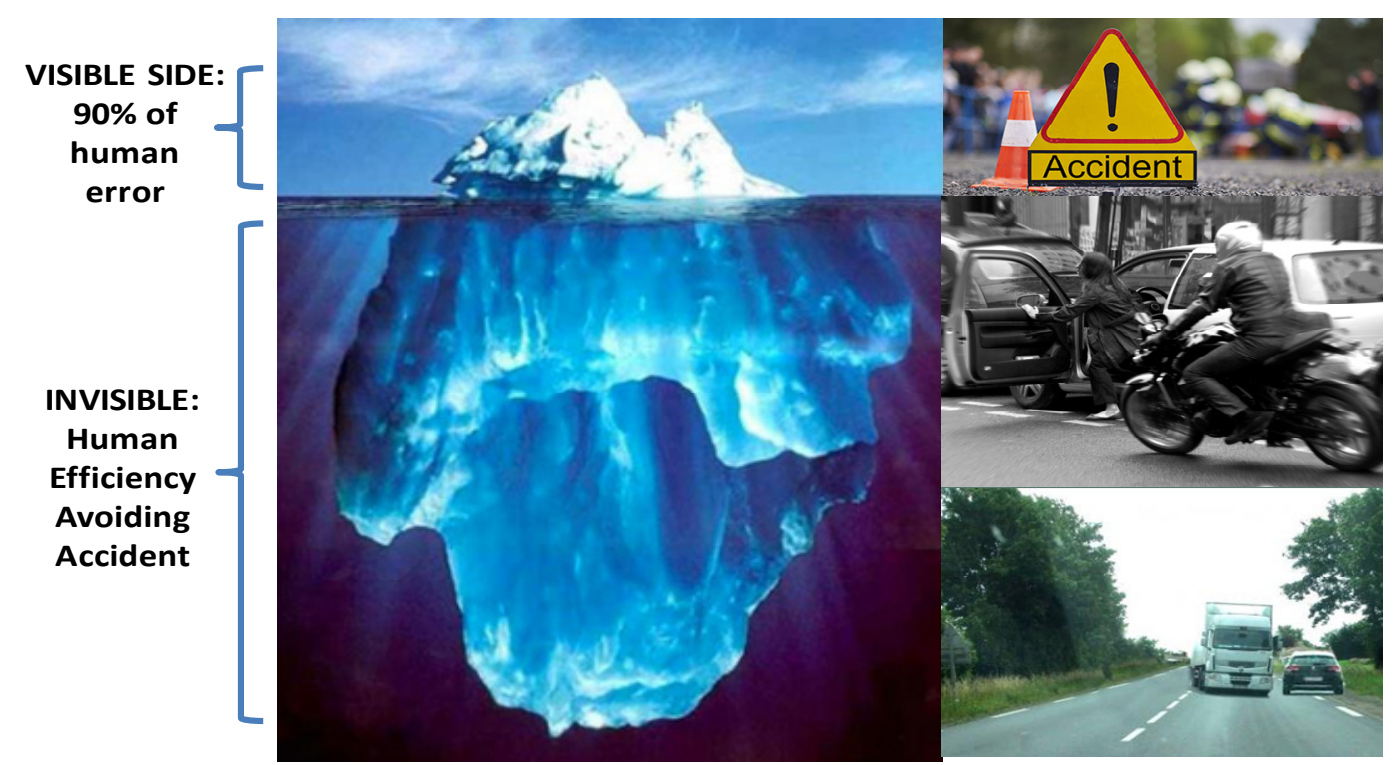

Fig. 2 Human efficiency hidden behind visible figures.

behind simplifying messages, sometimes amplified by press: Fig. 2 recalls that analyses are based on the visible side of accidentology, on the small percentage of cases when accident really occurred.

It is true that almost $90 \%$ of accidents are linked to human error, but it does not imply that this accident number will be reduced by ten with driver assistance or autonomous vehicles. When an accident occurs, the situation is then visible, but the main and invisible side is filled with risky situations where the accident has been avoided thanks to human reflex or appropriate arbitration.

With naturalistic driving experience like UDRIVE (European Naturalistic Driving and Riding for Infrastructure \& Vehicle Safety and Environment) [4], presented at NDRS2017 Congress (Naturalistic Driving Research) ${ }^{1}$, CEESAR is looking for risky situations, aiming to develop typology and models.

\section{Messages and HMI Standards}

The intermediate phase where most of connected vehicles are manually driven will probably last 20 or 30 years, as only $5 \%$ of the vehicles are renewed every year: the connected vehicle step has to be

\footnotetext{
1 NDRS 2017 Congress, Naturalistic Driving Research.
} http://ndrsymposium.com/. Updated July 1, 2017. efficient through messages to human drivers. The safety efficiency will result from a doubtful balance between pertinent alerts and driver distraction effects. In a risky situation, pertinent information can be efficient for most people, and a difficulty for some drivers.

This means that connected system will have:

(1) to transmit only relevant information to the driver;

(2) to alert the driver soberly, without stress addition;

(3) to guide to the best compromise with standard HMI.

In case the current HMI is not adapted to some drivers or very different from his usual vehicle, the road safety impact could be globally detrimental.

Fig. 3 shows that HMI can be very different and difficult to understand at first sight.

HMI standards are necessary to alert and guide drivers, and in addition, the transmitted messages cannot be limited to safety or navigation, as they have to guide to the best driving compromise: between ecology and safety, it can be a dilemma, for example, to decide how a descent can be exploited:

(1) to limit fuel consumption and emission, you could recommend to use the shortest but twisting road 


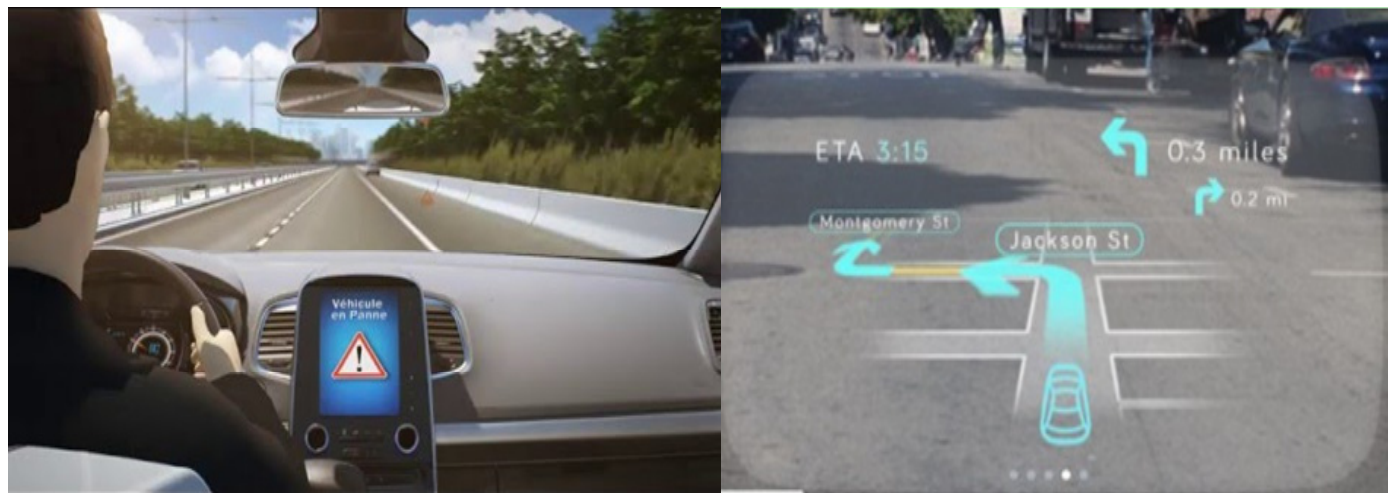

Fig. 3 HMI associating safety, traffic, and emissions.

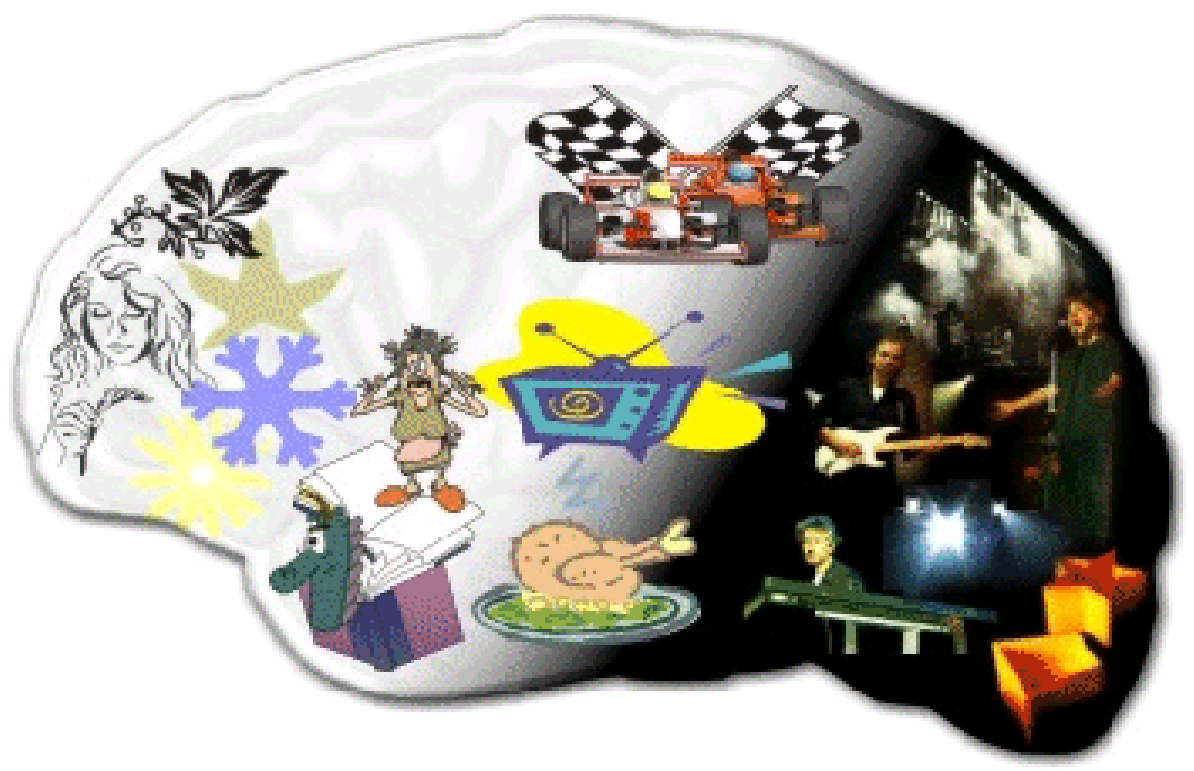

Fig. 4 Brain individual topics and arbitrations.

and to exploit the vehicle weight as an energy tank, which can be a safety concern;

(2) to maximize safety, a recommendation could be to brake at each road bend and to reaccelerate after, which is not the best for energy management and emissions.

The safety work has to be enlarged to emission and traffic specialists to reconcile ecology and safety.

Commonly, it will be necessary to anticipate situations between intelligent cars: it is theoretically feasible, but it is new and practically, some vehicles will not be connected (an example is two wheelers) and connected cars will still depend on a human driver.

\section{Human Behavior Management}

During the intermediate phase, the efficiency will depend on HMI standards, but also on human behavior.

As shown in Fig. 4, the brain complexity can manage very different subjects at the same time:

UDRIVE [4] project showed that car drivers are involved in distracting activities for $10 \%$ of the driving time, $20 \%$ for a truck driver. With human intelligence, a pertinent alert can be ignored, a clear recommendation can be circumvented, because of a personal concern or history, based on complex strategies which are difficult to analyze and simulate. 
Experimentation is ongoing with Renault and PSA vehicles from SCOOP@F [3] (French C-ITS project), which are equipped with a CEESAR [2] data recorder to analyze acceptability and efficiency when used by real drivers in real conditions. Doing so, the PSA and Renault Lab will get the first sight on the real HMI pertinence, in terms of acceptability and good usage.

In the future, it will have to be done at a larger scale, including all goals of driving recommendations, for traffic management, emission reduction and safety.

\section{Testing and Validations}

To take into account the huge diversity of human behavior, we have identified real risky situations, but we will have also to define and model typical situations to be managed in terms of traffic or emissions. A common benchmark of combinations between these situations will have to be simulated in front of real drivers, facing the proposed HMI.

To maximize the panel and get sufficient behavior representativity, virtual immersion has to be obtained at lower cost than today driving simulation means. Fig. 5 shows example where immediate interface like steering wheel is real with physical feedback, but where context situation is simulated in VR (virtual reality) glasses.

That implies developing such technology and sharing a typology of situations with technical standards, a lot of common work in front of us!

\section{Need to Work Together}

CEESAR can bring its 25 year experience of road safety improvement levers to develop such methodologies. Three domains have to be coordinated as shown in Fig. 6:

- First Domain: infrastructure adaptation, with public power and all road equipment companies;

- Second Domain: vehicle manufacturers and sale organizations to modernize the vehicle park;

- Third Domain: road user education based on potential messages and HMI standards.

With a pertinent connected mobility, it will be easier to offer driving delegation at an affordable price, a preliminary condition for a real deployment of autonomous vehicles: many societal improvements are associated with this target, which will also reveal

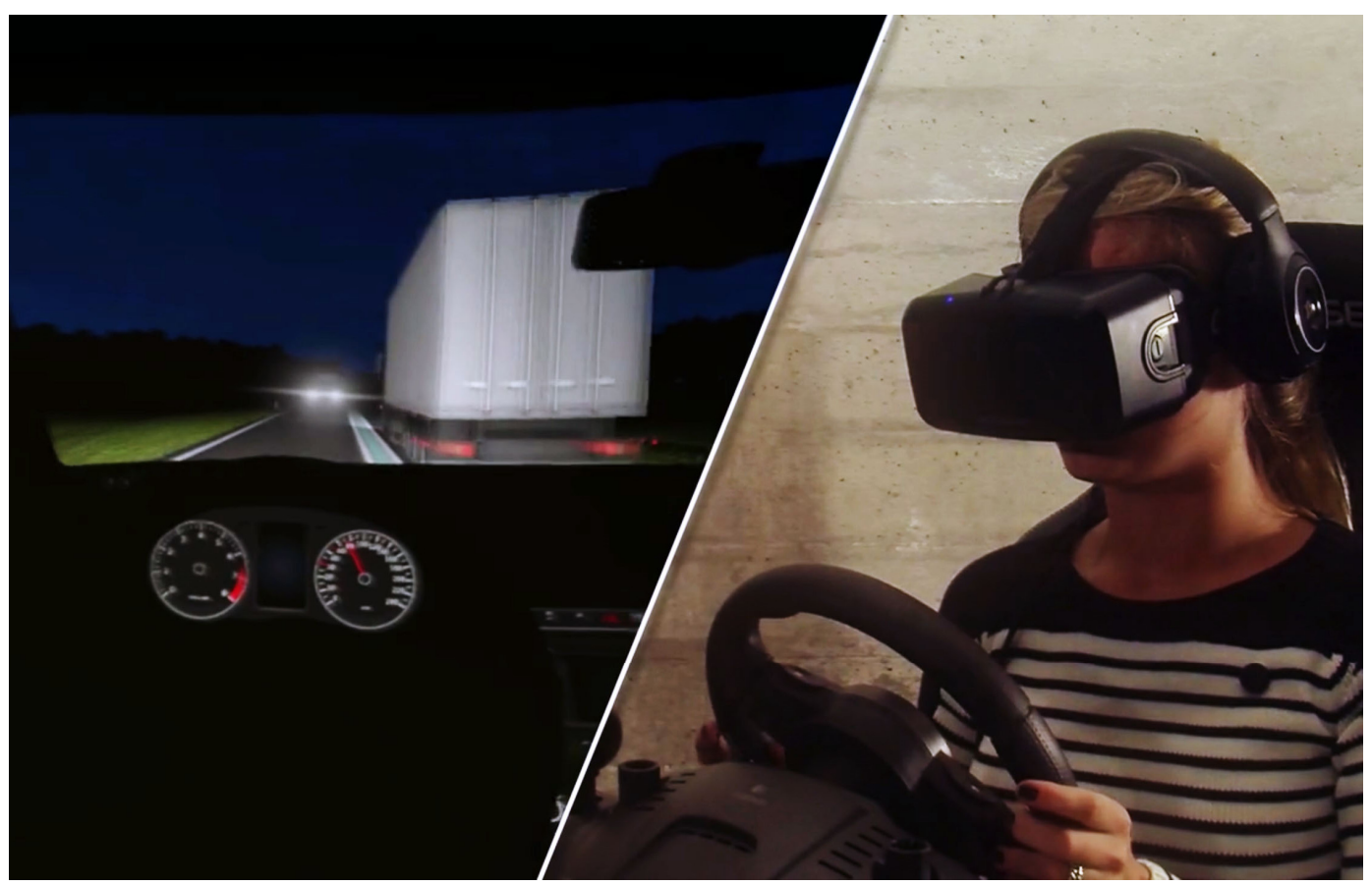

Fig. 5 Light testing using virtual reality glasses. 


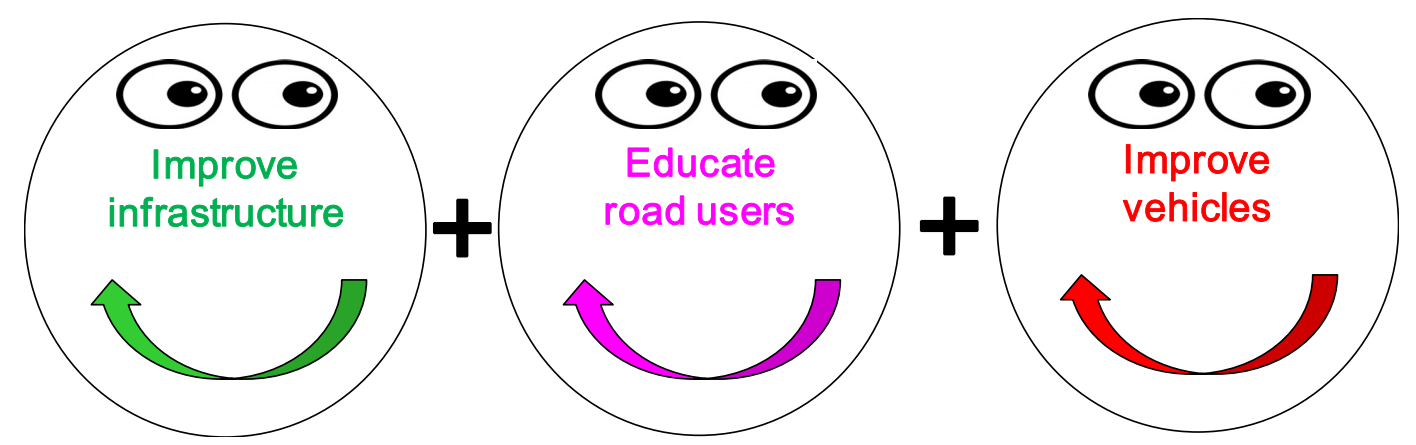

Fig. 6 Three domains to be coordinated.

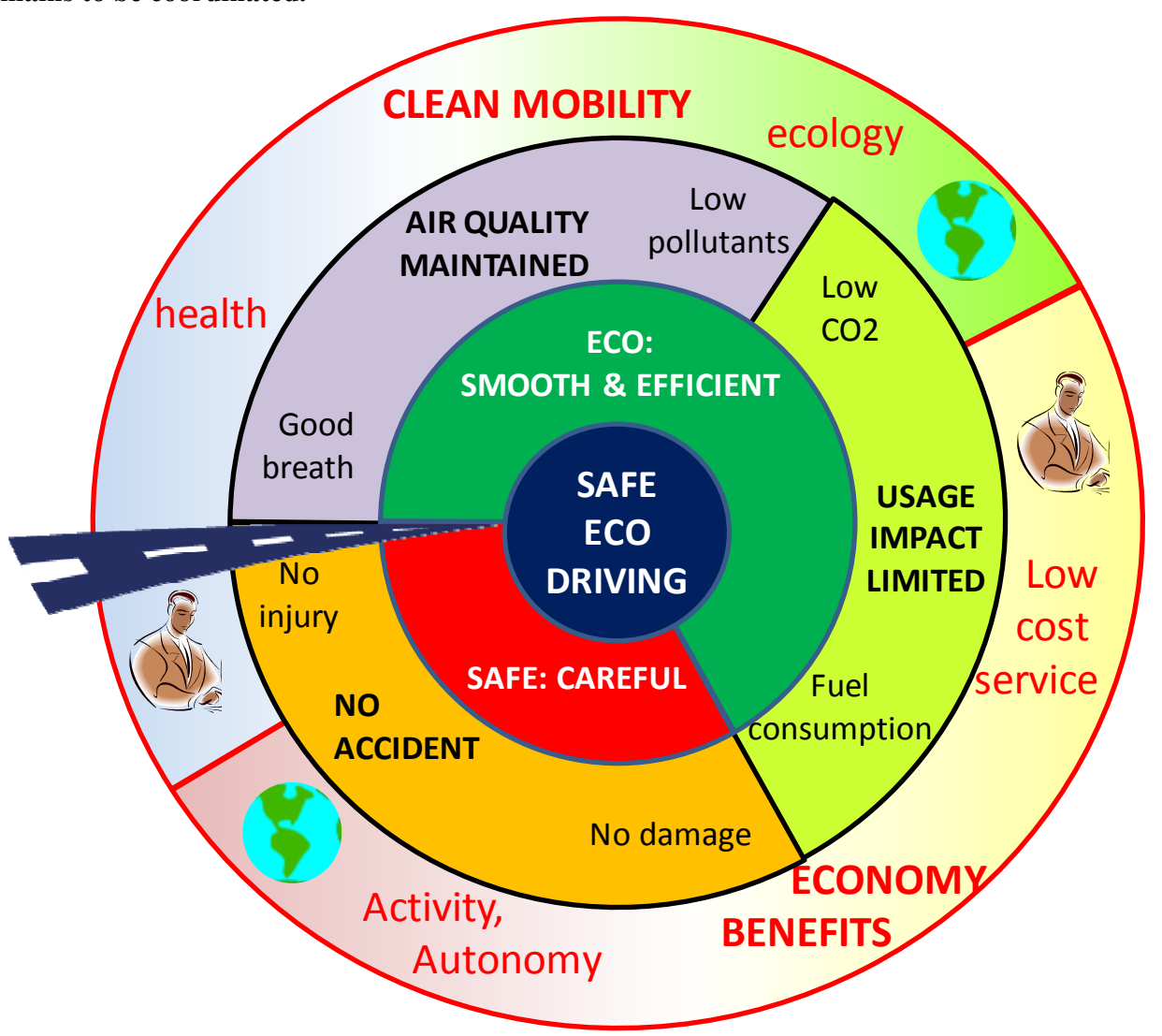

Fig. 7 Safe eco-driving for clean mobility and economy.

new safety issues to be solved, as passenger protection in positions which will be different from today. To demonstrate the safety and efficiency of these two steps, which are real automotive revolutions, it will be necessary to work all together.

With three research teams (accidentology data collection and statistics, crash biomechanics including PMHS, dummies and body numeric models, vehicle environment and human behavior), CEESAR already contributes to connected mobility and autonomous vehicle and is ready to work on future road safety with all involved partners.

\section{Conclusions}

Connected mobility is a major step to autonomous vehicle, to a mobility service which has to be efficient, safe and green at the same time.

As shown in Fig. 7, safe eco-driving means careful and smooth driving, efficient by anticipation of coming situations: this gives no accident and limited impacts, for clean mobility and economy benefits, at the individual level or worldwide societal level. 
The efficiency of the connected vehicle step is crucial for the second step, autonomous vehicle which is socially strategic. From now, it requires public funding and international authority to coordinate work, and fulfil four conditions:

- No societal efficiency without pertinent messages taking into account all targets: the alert and recommendations will have to improve safety, but also traffic and ecology, with aims to give time for activities, to reduce fuel consumption and vehicle emissions;

- No guarantee that messages are received if HMI principles are bad and different between brands: safety improvement has to include the shared vehicles market, where drivers are not familiar with the vehicle specificities;

- No hope that recommendations will be applied if they do not take into account driver interest: human behaviour is complex and individual, which means that a recommendation can circumvented;

- No confidence on efficiency without a global and representative validation: pilot studies are realized in France as SCOOP@F [3], but large virtual reality testing will be necessary before deployment, to confirm that connected mobility will really improve safety.

\section{References}

[1] ONISR. n.d. French Road Safety Organization. Data updated every month. Accessed March 1, 2017. http://www.securite-routiere.gouv.fr/la-securite-routiere/1 -observatoire-national-interministeriel-de-la-securite-rout iere/accidentalite-routiere.

[2] CEESAR. n.d. CEESAR website. Updated November, 2017. http://www.ceesar.fr.

[3] SCOOP@F Project. n.d. SCOOP@F Project Presentation. Accessed July 1, 2017. http://www.google. fr/url? $\mathrm{sa}=\mathrm{t} \& \mathrm{rct}=\mathrm{j} \& \mathrm{q}=\& \mathrm{esrc}=\mathrm{s} \&$ source $=$ web\& $\mathrm{cd}=2 \& \mathrm{cad}=$ rja\&uact $=8 \&$ ved $=0$ ahUKEwiHutPQp-rVAhVFtBoKHXk 9A0kQFggvMAE\&url=http $\% 3 \mathrm{~A} \% 2 \mathrm{~F} \% 2 \mathrm{Fwww}$.scoop.de veloppement-durable.gouv.fr $\% 2 \mathrm{Fen} \% 2 \mathrm{Fgeneral}$-presenta tion-a9.html\&usg=AFQjCNG44VEmC2vZTVLdGLN7J1 aV6o1vDQ.

[4] UDRIVE Project. n.d. European Naturalistic Driving Study. Accessed July 1, 2017. http://results.udrive.eu/. 